\title{
OBSERVATION DES NÉBULEUSES PLANÉTAIRES DANS L'INFRAROUGE
}

\author{
YVETTE ANDRILlat \\ (Université de Montpellier, France)
}

La région de l'infrarouge a été fort peu étudiée jusqu'à maintenant en astrophysique. En ce qui concerne les nébuleuses planétaires les seuls travaux publiés concernent la région de l'infrarouge photographique jusqu'à $1 \cdot 2 \mu$.

Au delà de cette limite, on se heurte à de grosses difficultés dues à la présence de l'atmosphère terrestre et au manque de détecteurs sensibles. Cependant, depuis 5 ans environ, un important effort technique a été réalisé principalement aux U.S.A. à l'Université d'Arizona, à San Diego et à California Institute of Technology par Low et Murray et Wildey (1963) pour mettre au point des cellules photoconductrices dopées permettant d'atteindre $15 \mu$. Low a déjà observé quelques nébuleuses planétaires dans cette région (non publié) et un vaste programme d'observation a été mis au point par Gillet, Stein et Low. Sur le plan théorique, de nombreux et remarquables travaux ont été menés à bien par Delmer et al. (1967).

Ce sont les études théoriques de Burbidge et al. (1963) qui montrèrent que, dans les régions $\mathrm{HII}$, une grande partie de l'énergie était émise sous forme de rayonnement infrarouge. Dès lors, il a paru intéressant d'étudier les nébuleuses planétaires dans cette région spectrale pour les raisons suivantes:

(1) Dans le domaine optique, l'absorption interstellaire empêche les observations à de grandes distances dans le plan galactique et au voisinage du centre galactique. Or, aux grandes longueurs d'onde, cette absorption est considérablement diminuée. En infrarouge, il est donc possible d'observer les nombreuses nébuleuses planétaires situées près du centre de la galaxie.

(2) Les raies infrarouges correspondant à des transitions interdites, le phénomène de self-absorption est négligeable. Elles fourniront donc des images monochromatiques permettant de préciser la structure de la nébuleuse.

(3) Des intensités des raies infrarouges, on déduit l'abondance des éléments. Dans les nébuleuses planétaires, la plupart d'entre eux sont présents dans plusieurs états d'ionisation qui ne sont pas tous observables dans le domaine optique. C'est p. ex. le cas du néon pour lequel NeII n'a pas de raies visibles dans le domaine optique. Par contre, une raie est observable dans l'infrarouge. La mesure de son intensité permettra d'accéder à l'abondance du néon.

(4) On trouve dans l'infrarouge quelques couples de raies d'un même élément qui 
sont désexcitées par collisions à des densités moyennes (comme 3727-3729 $\AA$ de [OII] p. ex.). Le rapport de leurs intensités fournit la densité électronique.

Les raies infrarouges considérées dans les calculs des spectres théoriques sont les raies les plus intenses relatives à certains ions particulièrement abondants dans les nébuleuses planétaires. Elles résultent de transitions entre les niveaux de structure fine des termes de l'état fondamental. Ces transitions interdites sont de type magnétique dipolaire correspondant à $\Delta J= \pm 1$, les transitions quadrupolaires, beaucoup plus faibles, n'ont pas été envisagées. Les différences d'énergie $\Delta E$ entre les niveaux de structure fine varient de $0 \cdot 01 \mathrm{à} 0 \cdot 1 \mathrm{eV}$ si bien que le mécanisme principal d'excitation des niveaux supérieurs est celui des collisions inélastiques d'électrons: en effet, pour les nébuleuses, les énergies des électrons incidents sont voisines de $1 \mathrm{eV}$, quantité nettement supérieure à $\Delta E$.

Delmer et al. (1967) ont calculé les intensités de ces raies infrarouges en utilisant les données déduites des observations dans le domaine optique. Ces intensités peuvent être calculées à partir de l'abondance de l'ion et de la force de collision $\Omega$ nécessaire pour exciter les niveaux supérieurs. Dans le cas des niveaux à structure fine, $\Omega$ est calculable par la 'Quantum Defect Method'. De nombreuses valeurs ont d'ailleurs été empruntées aux travaux de Seaton (1958), Osterbrock $(1964,1965)$ et Blaha (1964). Dans quelques cas, l'abondance peut être déduite de l'intensité des raies interdites optiques du même ion: on a alors de bonnes valeurs pour les intensités: c'est le cas notamment de SiII, Nev, AiII et Av.

En général, la détermination de l'abondance est très imprécise car il y a de grosses erreurs sur les constantes atomiques utilisées, en particulier sur les 'Cross-Section' de photo-ionisation dont les calculs sont complexes. Les valeurs données par Seaton (1958), et Ditchburn et Öpik (1962) ont été adoptées. Delmer et al. (1967) ont étendu les calculs aux ions complexes en utilisant la 'Quantum Defect Method' indiquée par Burgess et Seaton (1960), mais les résultats obtenus sont peu précis. L'imprécision dans la détermination des intensités peut atteindre un facteur 30 .

Les intensités ont été calculées pour les raies situées dans les 'fenêtres' de l'atmosphère.

Dans l'infrarouge, l'absorption atmosphérique est très importante: elle est due principalement aux nombreuses et très larges bandes de la vapeur d'eau. On trouve également les absorptions plus discrètes mais intenses de $\mathrm{CO}_{2}$ et enfin celles plus faibles de $\mathrm{N}_{2} \mathrm{O}, \mathrm{CH}_{4}, \mathrm{O}_{2}$ et $\mathrm{O}_{3}$ (Allen, 1964). Jusqu'à $2 \cdot 4 \mu$ environ, il existe plusieurs zônes ou 'fenêtres' dans lesquelles l'absorption atmosphérique est faible. Au delà, ces 'fenêtres' sont rares et s'étendent approximativement de 3.2 à $4 \cdot 1 \mu$, de 4.5 à $5.2 \mu$, de 8 à $13 \cdot 5 \mu$, et de 17 à $25 \mu$. Aux environs de $4 \cdot 2 \mu, 6 \cdot 5-15 \mu$ et au delà de $25 \mu$, l'atmosphère est à peu près opaque.

De plus, la présence d'une émission thermique de l'atmosphère perturbe fortement les mesures de photométrie photoélectrique surtout au delà de $10 \mu$.

Pour obtenir les intensités observées, il faut corriger les intensités calculées de 
l'absorption atmosphérique. Théoriquement, le problème est difficile à cause de la complexité des bandes de rotation-vibration de $\mathrm{H}_{2} \mathrm{O}, \mathrm{CO}_{2}$. Par ailleurs, les résultats expérimentaux manquent de précision car la distribution de la vapeur d'eau dans l'atmosphère n'est pas uniforme et l'absorption varie très rapidement d'un instant à l'autre. Les valeurs adoptées pour la transmission atmosphérique $T$ sont celles déduites des meilleurs travaux expérimentaux. Au zénith, elles sont approximativement de $T=0.08$ pour $\lambda=4.5 \mu ; 0.30$ pour $\lambda=7.9 \mu ; 0.90$ de 9 à $12.8 \mu ; 0.33$ pour $\lambda=13.1 \mu ; 0.38$ pour $\lambda=18.7 \mu ; 0.02$ pour $21.8 \mu ; 0.07$ pour $\lambda=24.2 \mu$; et 0.02 pour $\lambda=25.9 \mu$ (Delmer et al., 1967).

Les intensités $I$ ont été calculées pour 11 raies observables au-dessous de l'atmosphère. On a considéré seulement celles pour lesquelles $I$ est supérieur à $10^{-18}$ watts/ $\mathrm{cm}^{2}$.

\begin{tabular}{|c|c|}
\hline $\begin{array}{l}{[\mathrm{MgIV}]: \text { transition } J_{1}-J_{2}} \\
{[\mathrm{~A} \vee \mathrm{II}]}\end{array}$ & $\begin{array}{l}1 / 2-3 / 2 \text { à } \lambda=4.492 \mu . \\
3 / 2-1 / 2 \text { à } \lambda=4.525 \mu .\end{array}$ \\
\hline$[\mathrm{A} v]$ & $2-1 \quad$ à $\lambda=7.893 \mu$. \\
\hline [A III] & $1-2 \quad$ à $\lambda=8.990 \mu$ \\
\hline [Siiv] & $3 / 2-1 / 2$ à $\lambda=10.53 \mu$. \\
\hline$[\mathrm{Ne} I I]$ & $1 / 2-3 / 2$ à $\lambda=12 \cdot 8$ \\
\hline$[\mathrm{A} v]$ & $1-0 \quad$ à $\lambda=13 \cdot 1$ \\
\hline [S III] & $2-1 \quad \grave{a} \lambda=18.68$ \\
\hline [A III] & $0-1 \quad$ à $\lambda=21 \cdot 8$ \\
\hline$[\mathrm{Nev}]$ & $1-0 \quad$ à $\lambda=24 \cdot 2$ \\
\hline [OIv] & $3 / 2-1 / 2$ à $\lambda=25.87$ \\
\hline
\end{tabular}

Les spectres théoriques ont ainsi été calculés pour les 9 nébuleuses planétaires les plus brillantes: NGC 7027, IC 418, NGC 6572, NGC 6543, NGC 7662, NGC 7009, NGC 6826, NGC 6210 et NGC 6720. Pour NGC 7027, on a pû tracer le spectre de 3 à $28 \mu$ : les raies d'émission sont nettement au-dessus du 'bremsstrahlung' continuum. Stein (1967) signale d'ailleurs que, dans cette nébuleuse, le continu serait observable.

Parmi les émissions indiquées ci-dessus, l'une d'entre elles, [NeII] à $12.8 \mu$ est extrêmement importante car elle permet de déterminer l'abondance totale du néon. Jusqu'ici, l'absence de raies visibles de NeII a empêché une évaluation précise de cette quantité. Située dans une région où l'atmosphère est à peu près transparente, elle est donc observable dans de bonnes conditions à partir du sol. Particulièrement intense dans les régions $\mathrm{H}$ II (Gould, 1964), elle est également forte dans les nébuleuses planétaires de faible excitation. Pour IC 418 , Low a mesuré entre 12.5 et $13.5 \mu$ un flux de $1 \times 10^{-16}$ watts $/ \mathrm{cm}^{2}$ en bon accord avec l'intensité théorique $0.6 \times 10^{-16}$ watts $/ \mathrm{cm}^{2}$ calculée par Gould (1966) pour [NeII] à $12 \cdot 8 \mu$.

En dehors des 'fenêtres' de l'atmosphère, il existe d'autres raies observables (Osterbrock, 1967; Gould, 1963). 


\begin{tabular}{|c|c|c|c|}
\hline [AII]: transition $J_{1}-J_{2}$ & $3 / 2-1 / 2$ & 2 à $\lambda=6.983$ & $33 \mu$ \\
\hline$[\mathrm{Nev}]$ & $1-2$ & à $\lambda=14 \cdot 33$ & $\mu$ \\
\hline$[\mathrm{Ne} I I I]$ & $2-1$ & à $\lambda=15 \cdot 4$ & \\
\hline$[\mathrm{S} \mathrm{III}]$ & $0-1$ & à $\lambda=33.6$ & \\
\hline [SiII] & $1 / 2-3 / 2$ & 2 à $\lambda=34 \cdot 8$ & \\
\hline$[\mathrm{Ne} I I I]$ & $1-0$ & à $\lambda=36 \cdot 1$ & \\
\hline [OIII] & $1-2$ & à $\lambda=51 \cdot 71$ & \\
\hline [NIII] & $1 / 2-3 / 2$ & $2 \mathrm{a} \lambda=57 \cdot 31$ & \\
\hline [OIII] & $0-1$ & à $\lambda=88 \cdot 18$ & \\
\hline [OIII] & $0-1$ & à $\lambda=113$ & \\
\hline [NII] & $1-2$ & à $\lambda=112$ & \\
\hline [CII] & $1 / 2-3 / 2$ & 2 à $\lambda=156$ & \\
\hline [NIII] & $1 / 2-3 / 2$ & à $\lambda=174$ & \\
\hline$\left[\mathrm{N}_{\mathrm{II}}\right]$ & $0-1$ & à $\lambda=204$ & \\
\hline [OIII] & $1-2$ & à $\lambda=307$ & \\
\hline
\end{tabular}

Leurs intensités ont été calculées par Osterbrock (1967) pour une température de $7500^{\circ}$ et en utilisant les forces de collision données principalement par Seaton (1958). Quelques-unes de ces transitions sont particulièrement intéressantes car elles sont fonction de la densité électronique.

D'après les travaux bien connus de Seaton (1954) et Seaton et Osterbrock (1957), la mesure du rapport d'intensité de 2 raies interdites d'un même ion est un bon critère de densité électronique $N_{\mathrm{e}}$. C'est la méthode utilisée dans le domaine optique pour déduire $N_{\mathrm{e}}$ du rapport d'intensité des 2 raies de [O II] à 3726 et $3729 \AA$ p. ex.

Dans l'infrarouge lointain, il existe des couples de raies semblables (Osterbrock, 1967). Pour les densités électroniques échelonnées de $10^{2}$ à $10^{3}$ électrons par $\mathrm{cm}^{3}$, le rapport d'intensité $R$ des 2 raies de [NII] à $122 \mu$ et $204 \mu$ varie très fortement de $2 \cdot 7$ à 7.5 environ. Au delà de $10^{3}$ électrons par $\mathrm{cm}^{3}$, ce rapport est peu sensible aux variations de $N_{\mathrm{e}}$. Au voisinage de $5 \times 10^{3}$, il est préférable d'utiliser celui des 2 raies de [OIII] à $113 \mu$ et $307 \mu$. Pour $N_{\mathrm{e}}$ de l'ordre de $10^{5}$ electrons par $\mathrm{cm}^{3}$ ce sont les transitions de [NeIII] à $15 \cdot 4 \mu$ et $36 \cdot 1 \mu$ qui seront employées.

Les observations dans l'infrarouge effectuées au sol permettront essentiellement de résoudre les problèmes d'abondance des éléments, en particulier celui très important de l'abondance du néon. Les observations faites au delà de notre atmosphère terrestre, complèteront les précédentes et de plus fourniront les densités électroniques des nébuleuses planétaires.

\section{Remercîments}

Je remercie vivement le Professor M.J. Seaton qui m'a communiqué très aimablement les 'Preprints' des travaux de Delmer et al. (1967) et de Osterbrock (1967). Que tous ces auteurs trouvent ici l'expression de ma profonde gratitude. 


\section{Bibliographie}

Allen, C.W. (1964) Astrophysical Quantities, 2e éd., The Athlone Press, pp. 125-126.

Blaha, M. (1964) Bull. astr. Inst. Csl., 15, 33.

Burbidge, G. R., Gould, R.J., Pottasch, S.R. (1963) Astrophys. J., 138, 945.

Burgess, A., Seaton, M.J. (1960) Mon. Not. R. astr. Soc., 120, 121.

Delmer, T.N., Gould, R.J., Ramsay, W. (1967) Astrophys. J., 149, 495.

Ditchburn, R.W., Öpik, U. (1962) dans Atomic and Molecular Processes, D. R. Bates (Red.), Academic Press, New York, p. 79.

Gould, R.J. (1963) Astrophys. J., 138, 1308.

Gould, R.J. (1964) Ann. Astrophys., 27, 815.

Gould, R.J. (1966) Astrophys. J., 143, 603.

Murray, B.C., Wildey, R.L. (1963) Astrophys. J., 137, 692.

Osterbrock, D.E. (1964) A. Rev. Astr. Astrophys., 2, 95.

Osterbrock, D.E. (1965) Astrophys. J., 142, 1423.

Osterbrock, D.E. (1967) Expected Infrared Spectra of Gaseous Nebulae. Presented at Royal Society Discussion Meeting on Infrared Astronomy, à paraître dans Phil. Trans. R. Soc. Lond. A, 'Preprint' en communication privée.

Seaton, M.J. (1954) Ann. Astrophys., 17, 296.

Seaton, M.J. (1958) Rev. Mod. Phys., 30, 379.

Seaton, M.J., Osterbrock, D.E. (1957) Astrophys. J., 125, 66.

Stein, W.A. (1967) Astrophys. J., 148, 295. 